\title{
Factors influencing long-term pessary use: comment
}

\author{
Shigeki Matsubara
}

Published online: 2 April 2011

(C) The International Urogynecological Association 2011

\section{Dear Editor,}

Friedman et al. [1] studied 150 women with pelvic organ prolapse using a pessary for over 1 year. They showed that while $115(77 \%)$ continued using pessaries after 1 year, the remaining 35 (23\%) discontinued. Reasons for discontinuation were surgery in 25 and pessary removal without further treatment in 10. I am wondering whether these 10 patients with pessary removal continued to have prolapse. Did prolapse disappear, or at least become less severe, in some of them? Was that why they received no further treatment?

To characterize patients with successful pessary fitting is clinically important. However, there may be another aspect beyond successful vs. unsuccessful fitting: A pessary may do more; it may reposition the uterus not only during implantation but also after its removal in some patients.

It is unfortunate that publication delay may have prevented Friedman et al. [1] from citing our recent article [2]. We showed that in six patients with complete uterine prolapse who were fitted with ring pessary, prolapse disappeared even after pessary removal. Median duration of pessary insertion was 27.5 (range 13-73) months, and the total observation time has a median of 78.0 (range 52-111) months. Longterm follow-up was needed to clarify this phenomenon. The

A reply to this comment can be found at doi:10.1007/s00192-011-1393-4.

S. Matsubara $(\bowtie)$

Department of Obstetrics and Gynecology,

Jichi Medical University,

3311-1 Shimotsuke,

Tochigi 329-0498, Japan

e-mail: matsushi@jichi.ac.jp importance of long-term follow-up was also described by Friedman et al. [1].

We had proposed two possible reasons why ring pessary, even after its removal, repositioned the uterus [2]. Pessary fitting may relieve stretching of the pelvic floor muscle, which may regain muscle strength. Alternatively, local inflammation cased by vaginal erosion may lead to vaginal fibrosis. This may prevent the uterus from descending; however, this remains a speculation.

My three decades of clinical observation have given me an impression that the required size of the ring pessary may decrease over time in some patients, especially in long-term pessary users. In some patients, the appropriate size may decrease until the pessary finally becomes unnecessary, as shown by us [2]. If the pessary remains in place for a long time without periodic check-up, it may become embedded in the site in some patients. A study focusing on changes over the time course, and not solely on successful vs. unsuccessful fitting, may contribute to a better understanding of the underlying mechanism of how a pessary cures prolapse.

Pessary may not be only palliative but also a therapeutic option for uterine prolapse in some patients. Further study in a larger population may be needed to clarify how and in whom this phenomenon, repositioning of the uterus after pessary removal, occurs.

\section{References}

1. Friedman S, Sandhu KS, Wang C, Mikhail MS, Banks E (2010) Factors influencing long-term pessary use. Int Urogynecol J Pelvic Floor Dysfunct 21:673-678. doi:10.1007/s00192-009-1080-x

2. Matsubara S, Ohki Y (2010) Can ring pessary have a lasting effect to reverse uterine prolapse even after its removal? J Obstet Gynaecol Res 36:459-461 